Bangladesh J Med Microbiol 2018; 12 (2): 1-3

Bangladesh Society of Medical Microbiologists

\title{
Editorial
}

\section{Biologics: A Therapy of New Era}

\section{S. M. Shamsuzzaman}

Professor \& Head, Department of Microbiology, Dhaka Medical College, Dhaka, Bangladesh.

'Biologics' or 'biological therapy' or 'biological drugs' are a class of immunosuppressive drugs, which include a wide variety of products derived from human, animal, or microorganisms produced by using Biotechnology. These are genetically engineered proteins that target specific parts of the immune system. Now a days, such drugs are being used to treat various autoimmune diseases or the diseases linked someway to immunological mechanisms that fails to improve with other drugs. They are difficult to make than conventional drugs so they are costlier.

Updated knowledge of the immune/inflammatory pathways as well as better understanding of the pathophysiology of autoimmune diseases including rheumatoid arthritis (RA) and seronegative spondyloarthropathies (such as ankylosing spondylitis and psoriatic arthritis), have provided the link between inflammation of bone and joints through a complex network of bone cells, T and B cells, pro-inflammatory cytokines (such as TNF-a, IL1, IL6, IL17, IL23), costimulator molecules, and signaling pathways.

The available data extracted from clinical and experimental studies, conducted with TNF-a, IL6 and IL1 blockers, as well as B and T cell therapies, have demonstrated a beneficial effect in reducing the symptoms and signs of inflammatory diseases. Biological therapy is being successfully used in multiple advanced malignancies in recent time.

Biologics are in widespread use in different clinical situations like, Rheumatoid arthritis (RA), Ankylosing spondylitis, Crohn's disease, Ulcerative colitis, Psoriasis, Diabetes, Multiple sclerosis (MS), various cancers.

Correspondence:

S. M. Shamsuzzaman

Professor \& Head

Department of Microbiology

Dhaka Medical College, Dhaka, Bangladesh.

Email: smszaman@yahoo.com
Allograft survival has been increased after incorporation of biologics in transplantation.

For RA, use of biologics has resulted in a therapeutic revolution in the treatment outcome where these medications are included as disease-modifying antirheumatic drugs (DMARDs). Biologics are proven to be more effective in a significant number of patients with RA who failed to achieve satisfactory response with other conventional DMARDs. For adult RA, nine biologics have been licensed until now including five TNF inhibitors (infliximab, etanercept, adalimumab, golimumab and certolizumab), rituximab, abatacept, tocilizumab and anakinra. Recently a new class of drugs, tyrosine kinase inhibitors, also known as 'directed synthetic DMARDS' (tofacitinib and baricitinib) have been approved for use in RA patients, in cases where conventional DMARD and/or biologics fail to achieve improvement.

Desirable therapeutic response to biologics has also been observed in the treatment of psoriatic arthritis, and other forms of inflammatory arthritis and thus biologics have become life-changing for many people.

But as most of the biologics suppresses immunity, so chance of new infection or reactivation of latent infection may occur. Others may have a higher risk for developing certain types of cancer. Long-term use in transplant patients has been associated with an increased risk of malignancy. Common side effects of biologic drugs include allergic reactions, injection site reactions, chills, weakness, diarrhea, nausea, vomiting, rash, sore throat. Serious side effects of biologic drugs include low blood pressure, anaphylaxis, and serious infections.

Categories of Biologics are i) B-cell inhibitors, ii) Interleukin inhibitors, iii) Selective Co-stimulation modulator, iv) Tumor necrosis factor inhibitors (TNF-Inhibitors). Each of the 4 classes of biologics is chosen for its unique inflammatory target. Each has beneficial effect as well as risks. Biologics are given as an injection or infusion. 
Tumor Necrosis Factor-a Inhibitors: Examples: adalimumab, certolizumab, etanercept, golimumab, and infliximab.

Uses: These drugs reduce inflammation and limit disease progression in inflammatory/autoimmune diseases. They are sometimes used in combination with other medications. The patient may notice an improvement of the symptoms within 2 or 3 doses. Etanercept, adalimumab and infliximab are approved for use in children; the only TNF inhibitor that can be used during pregnancy is 'certolizumab'.

Side effects: There is an increased risk of tuberculosis and fungal infections. The risk of tuberculosis may be lower with 'etanercept' than other TNF inhibitors. Long-term use of these medications increases the risk of certain cancers. Adalimumab may worsen pre-existing heart failure or multiple sclerosis. Antibodies are formed against the drug and the anti-inflammatory effect of anti-TNF drugs can be reduced over time; using methotrexate with the biologic may reduce it.

B-Cell Inhibitors: (Examples: belimumab and rituximab)

Uses: B-cell inhibitors are often used to treat RA when other treatments are not effective. Use of 'rituximab', a chimeric monoclonal immunoglobulin $G$ antibody directed against CD20, showed a promising result in the treatment of refractory autoimmune bullous disease (pemphigus and pemphigoid) in a multicenter study . The effects of rituximab treatment (two infusions approximately two weeks apart) can keep a patient symptom free for about one year.

Side effects: The infusion itself can have risks, such as a change in blood pressure, chest pain, difficulty in breathing, rash, dizziness and/or flu-like symptoms. Medications can be given before the infusion to prevent these reactions. The patient may be more susceptible to getting colds or sinus infections after receiving it.

Interleukin Inhibitors: (Examples: anakinra, tocilizumab, canakinumab, secukinumab, ustekinumab, ixekizumab, and sarilumab).

Uses: Interleukin inhibitors can be used for cases who don't find relief from TNF inhibitors,. This class of drugs is effective and well tolerated by most people.

Side effects: Intestinal perforation may occur rarely. The patient should be aware of changes in body temperature, abdominal discomfort, unusual bowel movements, headaches or symptoms of infection.
Selective Co-stimulation Modulators: (Example: abatacept)

Uses: Abatacept is usually used if other DMARDs (e,g. methotrexate) do not work well; it can also be used as a first-line treatment for moderate to severe RA. Some people who do not get relief from an anti-TNF respond to abatacept. To treat juvenile idiopathic arthritis, it is one of the commonly used biologics. After 4-6 weeks of receiving treatment, stiffness, joint pain and swelling may be relieved. For observing maximum response, 4-6 months of treatment with abatacept is necessary. Combined therapy of abatacept with methotrexate is more effective than methotrexate alone. It has few interactions with other drugs.

Side effects: Patients receiving abatacept may develop pneumonia, tuberculosis and influenza. Cough, sore throat, headache and nausea may occur in addition to infusion reaction which is relatively more common with treatment with abatacept.

Combination of biologics with other drugs: Biologics are used singly or in combination with other non-biologic drugs. Methotrexate is often combined with a biologic in reducing inflammatory diseases. It is to remember that combining biologics doesn't always be beneficial, it can increase risks.

Chance of infection is more among the patients receiving biologics: The patient receiving biologics are more prone to develop infection because biologic therapies suppress the immune system. The most common are colds, upper respiratory tract infection, sinus infection, sore throat, bronchitis or urinary tract infections.

To avoid getting infection the patients receiving biologics should follow the following: 1) washing hand frequently; 2) avoiding crowded areas, enclosed spaces, public transportation and childcare facilities; 3) informing friends and family not to visit if they are sick; 4) talking to doctor before scheduling vaccinations, dental appointments or manicures, 5) should not share cups, utensils or personal items; 6) should not swim in lakes, rivers, ponds or public pools and should avoid hot tubs; 7) should not come in contact with pets; 8) birds, fish, rodents, reptiles and farm animals should be avoided; 9) it is not safe to work in the garden with soil or mulch; 10) avoiding unpasteurized food, raw eggs or fish, soft cheeses and shellfish.

\section{Precautions before taking biologics}

Treatment with biologics should not be started if the patient has recent infection or have chronic infection of 
any kind. In case of acute infection the patient should be treated with antibiotic and biologics should not be started until the infection is cleared off. As more than $90 \%$ tuberculosis occur due to reactivation of latent infection and biologics can reactivate the infection, so tuberculin test or blood test for TB (e.g. Quantiferon Gamma assay) should be done before starting any biologic. Reactivation of Hepatitis viruses may occur with any biologic, so $\mathrm{HBsAg}$ and test for $\mathrm{HCV}$ are also needed.

If a patient has co morbidities like cancer, diabetes, a heart disease or neurological disorders, he is in increased risk of getting infection and the patients should be aware about it.

Should be careful about vaccinations: The patient on biologics should not take any live vaccines; if it is needed it should be taken one to three months before starting biological drugs. However, inactivated vaccines can be administered at any time.

Avoidance of surgery: Any type of surgery should be avoided during biological therapy. Therapy should be stopped if any surgery is done and should restart the biologic only after the sutures are removed and in absence of any sign of infection. Long-term safety studies for fetuses and infants have not been determined for most of the available biologics.

In case of hypersensitivity to latex or rubber, 'adalimumab' should not be prescribed since the prefilled syringe contains these. If a patient recently takes 'etanercept' or if a patient is allergic to Escherechia coli proteins, he should not take anakinra because these can cause adverse reactions in some patients.

\section{Further Reading}

Boutros C, Tarhini A, Routier E, et al. Safety profiles of anti-CTLA-4 and anti-PD-1 antibodies alone and in combination. Nat Rev Clin Oncol 2016; 13(8):473-86. doi: 10.1038/nrclinonc.2016.58.

Di Munno O, Ferro F. The effect of biologic agents on bone homeostasis in chronic inflammatory rheumatic diseases. Clin Exp Rheumatol 2019; 37(3): 502-507.
Kurihara Y, Yamagami J, Funakoshi T, et al. Rituximab therapy for refractory autoimmune bullous diseases: A multicenter, open-label, single-arm, phase $1 / 2$ study on 10 Japanese patients. J Dermatol, First published: 26 December 2018. https://doi.org/ 10.1111/1346-8138. 14732.

Kang S, Tanaka T, Kishimoto T. Therapeutic uses of anti-interleukin-6 receptor antibody. Int Immunol 2015; 27(1): 21-29.

Kivitz A, Olech E, Borofsky M, et al. Subcutaneous tocilizumab versus placebo in combination with disease-modifying antirheumatic drugs in patients with rheumatoid arthritis. Arthritis Care Res 2014; 66(11): 1653-1661.

Levine O, Devji T, Xie F. A new frontier in treatment of advanced melanoma: Redefining clinical management in the era of immune checkpoint inhibitors. Hum Vaccin Immunother 2017; 13(8):1765-1767. doi: 10.1080/ 21645515.2017.1322241.

Sanmart R, Ruiz-Esquide V, Bastida C, Soy D. Tocilizumab in the treatment of adult rheumatoid arthritis. Immunotherapy 2018; 10(6): 447-464.

Smolen JS, Landewe R, Bijlsma J, Burmester G, Chatzidionysiou $\mathrm{K}$, Dougados $\mathrm{M}$ et al. EULAR recommendations for the management of rheumatoid arthritis with synthetic and biological disease-modifying antirheumatic drugs: 2016 update. Ann Rheum Dis 2017; 76(6): 960-977.

Strand V, Burmester GR, Ogale S, Devenport J, John A, Emery P. Improvements in health-related quality of life after treatment with tocilizumab in patients with rheumatoid arthritis refractory to tumour necrosis factor inhibitors: results from the 24-week randomized controlled RADIATE study. Rheumatology 2012; 51(10):1860-1869.

Winthrop KL. The emerging safety profile of JAK inhibitors in rheumatic disease. Nat Rev Rheumatol 2017; 13(4): 234-243. 